\title{
A PROSPECTIVE NON-RANDOMISED OBSERVATIONAL CASE STUDY- THE STUDY OF OTOMYCOSIS
}

\author{
S. Senthil Kumaran ${ }^{1}$, M. Kavitha ${ }^{2}$
}

${ }^{1}$ Senior Assistant Professor, Department of ENT, Government Dharmapuri Medical College Hospital, Dharmapuri. ${ }^{2}$ Assistant Professor, Department of ENT, Government Dharmapuri Medical College Hospital, Dharmapuri.

\section{ABSTRACT}

\section{BACKGROUND}

Otomycosis is one of the most common cases which the otorhinolaryngologist faces in his day-to-day practice. Otomycosis is often associated with other comorbid conditions like AOM, CSOM, OE, etc. Latest knowledge in otomycosis will update the otorhinolaryngologist in providing better care to their patients.

\section{MATERIALS AND METHODS}

The study group consisted of fifty [50] patients of various age groups of both sexes. The samples were collected using sterile swabs, placed in test tube and then transported for smear and culture study. Microscopic examination is done and the second swab was inoculated onto media. Irrespective of culture results, $1 \%$ clotrimazole ear drops- 4 drops, 8 hourly for four weeks was tried and the patients were periodically examined for clearance of infection.

\section{RESULTS}

Most of the patients presented in their $3^{\text {rd }}$ and $4^{\text {th }}$ decade, majority being females. Aspergillus niger is the commonest fungal isolate. Ear probing is the most common presenting factor. Unilateral itching and blocking sensation is the usual presentation.

\section{CONCLUSION}

In this study, the most common offending fungus causing otomycosis is Aspergillus niger [46\%]. The most common presenting symptom is itching. Prolonged use of antibiotic or steroid drops and ear probing is the commonest predisposing factor. 1\% clotrimazole ear drops was effective in most cases [96\%].

\section{KEYWORDS}

Otomycosis, Fungal Lesions, Otitis Externa, KOH Mount, 1\% Clotrimazole Ear Drops, Aspergillus Niger.

HOW TO CITE THIS ARTICLE: Kumaran SS, Kavitha M. A prospective non-randomised observational case study- The study of otomycosis. J. Evolution Med. Dent. Sci. 2017;6(12):931-935, DOI: 10.14260/Jemds/2017/199

\section{BACKGROUND}

The ear is continually subjected to challenge by a variety of fungi, bacteria, yeasts and other microorganisms that are present in the external environment. Accumulation of debris, particularly in damp topical environment allows colonisation and infection of the ear. The majority of fungal organisms involved are soil saprophytes whose airborne conidia find the injured tissue a suitable environment for growth. Therefore, the mycology involved in ear is quite different from that encountered in systemic or cutaneous infections. Otomycosis is worldwide in distribution and in various recorded series has accounted for 5 to $20 \%$ of all cases of infective otitis externa. ${ }^{1}$ It is one of the commonest manifestations in India during rainy seasons. It occurs mostly in humid atmosphere. Otitis externa due to fungal infection may resemble the desquamative form of diffuse infective otitis externa. ${ }^{2}$

The incidence of fungal lesions of the ear has increased largely. This may be because of the underlying predisposing factors like immunocompromised situations such as use of

Financial or Other, Competing Interest: None.

Submission 30-12-2016, Peer Review 21-01-2017,

Acceptance 30-01-2017, Published 09-02-2017.

Corresponding Author:

Dr. S. Senthil Kumaran,

Senior Assistant Professor,

Department of ENT,

Government Dharmapuri Medical College Hospital,

Dharmapuri-636701.

E-mail:drsskent@gmail.com

DOI: $10.14260 /$ jemds $/ 2017 / 199$ antimicrobial, immunosuppressive and anticancer agents, transplantations, endocrinal disorders, etc.

Hence, by increasing awareness about mycotic lesions of ear, it will help for an early diagnosis and prompt treatment of life threatening fungal infections. Moreover, in the recent years "opportunistic fungal infections" have emerged as another important issue due to several contributory factors. This calls for increased awareness and definitive diagnosis.

With the above views in mind this study was undertaken to find out the different fungi that cause otomycosis and the clinical response to topical clotrimazole. A total number of 50 patients were examined and investigated for this case study.

\section{Aims of the Study}

1. To study the pattern of fungal infections in patients suspected of having otomycosis.

2. To identify the predisposing factors involved in the causation of mycotic ear infections.

3. To evaluate the different symptomatology with which a patient with fungal lesion of ear is presenting.

4. To study the response of the antifungal agent (topical clotrimazole) in patients with otomycosis.

\section{MATERIALS AND METHODS}

\section{Selection of Study Group}

The study group consisted of fifty (50) patients of various age groups of both sexes.

Materials for this study were collected from patients attending the ENT Outpatient Department, Upgraded 
Institute of Oto-Rhino-Laryngology, Madras Medical College, Chennai. Patients presenting with symptoms and signs of otomycosis, viz., ear pain, pruritus, ear discharge, fungal debris in the external canal were included in the study.

\section{Method of Collection of Specimen}

The samples were collected under aseptic precautions taking care to avoid contaminations. Sterile swabs were heavily charged with the suspected, fungal debris in the external auditory canal by repeatedly rubbing them firmly with the walls of the canal and placing them inside the sterile test tubes immediately. The test tube is tightly closed and wrapped in sterile polythene bag. Swabs were taken separately for smear and culture study.
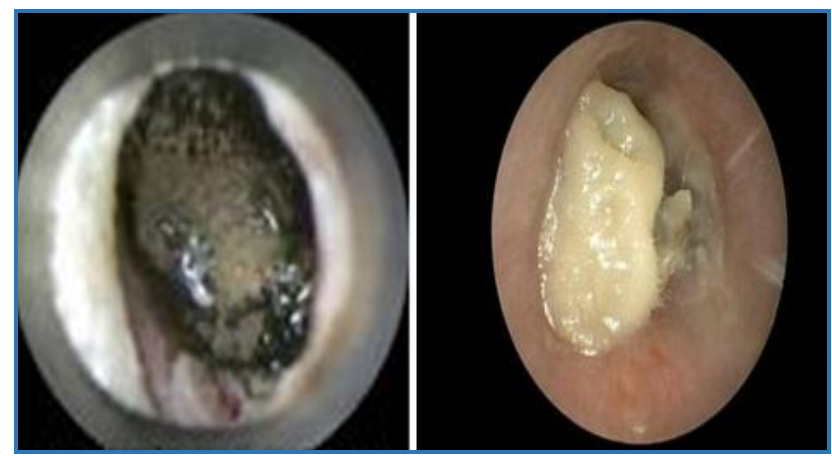

Figure 1. Otoendoscopic Appearance of Fungal Flakes

\section{Transportation of Specimens}

In order to avoid delay in collection of specimens and their transportation to the working place, the patients selected after taking the appropriate clinical examination and filling up of the 'Proforma' were made to wait till the last case was examined at O.P.D. Then specimens were collected and transported. All the specimens were given to the Mycology Laboratory, The Institute of Microbiology, Madras Medical College, Chennai and processed immediately.

\section{Processing of Specimens}

Microscopic Examination

Direct smear prepared was stained by Gram's stain technique and examined under oil immersion.

A wet mount was prepared in $10 \% \mathrm{KOH}$ solution for microscopic examination and Direct Lactophenol Cotton Blue Mount was done.

The KOH mount was kept at room temperature for 15 minutes and examined under low and high power and observed for the presence of septate/non-septate hyphae and structures like yeast cells and spores of fungi.

\section{Inoculation of Media}

The material on the second swab was inoculated onto Sabouraud dextrose Agar slant without antibiotic. It was then set at room temperature.

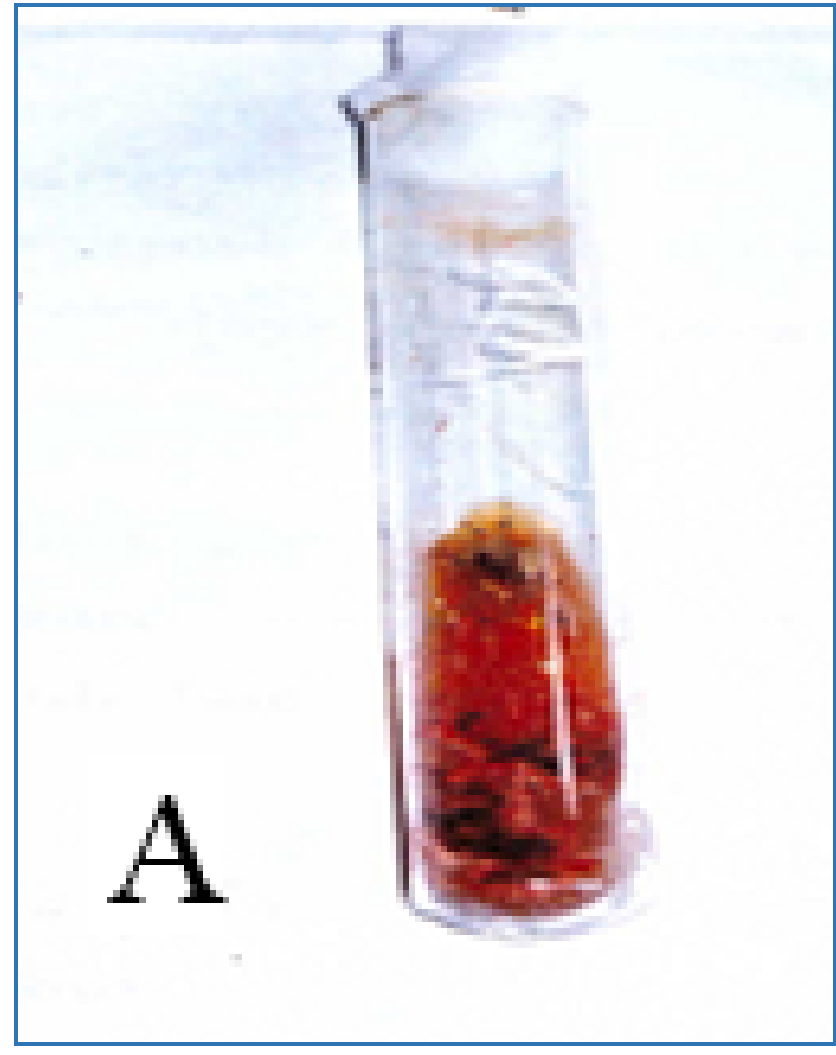

Figure 2A. Cephalosporium in Sabouraud Dextrose Agar Slant

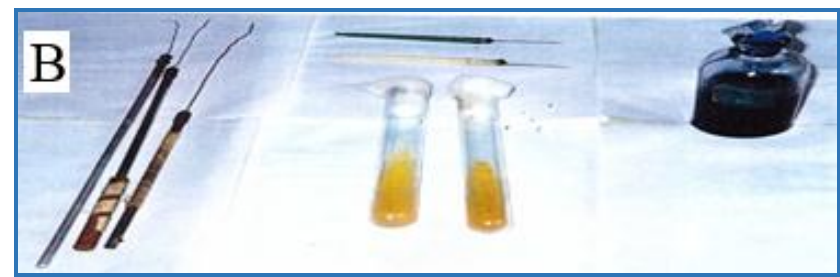

Figure 2B. Materials in Mycology Work

\section{Examination of Cultures}

The Sabouraud Dextrose Agar slants were examined after 2 days, 5 days, 7 days, 14 days and 21 days for the presence of growth. When growth occurred, it was identified by standard.

\section{Procedures used for Fungal Cultures}

In the case of candida, germ tube test was performed to know the pathogenicity. Reports were given after macroscopic and microscopic examination of culture with Lactophenol Cotton Blue Mount and Gram's Stain Smear study.

Irrespective of the culture results, $1 \%$ clotrimazole (Candid) ear drops- 4 drops* 8 hourly for four weeks was tried since culture results take some time. Patients were examined at weekly intervals. Where fungal infection persisted, the treatment was repeated. Patients presenting with severe earache due to canal wall oedema were treated initially with Icthammol-glycerine packing daily for 4 days and anti-inflammatory drugs orally. In some cases, syringing was done to remove the fungal mass.

\section{RESULTS}

The results on the study of otomycosis were as follows:

Age distribution of the study group with otomycosis showed age distribution from 7 to 70 years with predominant 
females. Average age of presentation was 34 years. Most of the patients were in their third and fourth decade.

Table 1. Shows the age distribution of the patients.

Table 2. Shows the percentage of sex distribution.

\begin{tabular}{|c|c|c|}
\hline Years & No. of Patients & Percentage \\
\hline 0 to 10 & 1 & 2 \\
\hline 11 to 20 & 6 & 12 \\
\hline 21 to 30 & 12 & 24 \\
\hline 31 to 40 & 13 & 26 \\
\hline 41 to 50 & 13 & 26 \\
\hline 51 to 60 & 2 & 4 \\
\hline 61 to 70 & 1 & 4 \\
\hline 71 to 80 & \multicolumn{3}{|c|}{ Table 1. Age Distribution } \\
\hline \multicolumn{3}{|c|}{} \\
\hline
\end{tabular}

\begin{tabular}{|c|c|c|}
\hline Sex & No. of Patients & \% \\
\hline Males & 19 & $38 \%$ \\
\hline Females & 31 & $62 \%$ \\
\hline \multicolumn{3}{|c|}{ Table 2. Sex Distribution } \\
\hline
\end{tabular}

Vast majority of the study group patients with otomycosis presented with unilateral disease irrespective of the predisposing factors which was illustrated in the following table.

\begin{tabular}{|c|c|c|}
\hline Laterality & No. of Patients & $\mathbf{\%}$ \\
\hline Unilateral & 45 & 90 \\
\hline Bilateral & 5 & 10 \\
\hline \multicolumn{2}{|c|}{ Table 3. Laterality } \\
\hline
\end{tabular}

Regarding the symptoms of patients with otomycosis, the most common symptom was itching of ears followed by blocking sensation, ear pain, tinnitus and watery discharge in that order.

Table 4 shows the various symptoms and their incidence.

\begin{tabular}{|c|c|c|}
\hline Aural Symptoms & No. of Patients & Percentage \\
\hline Pain & 36 & 72 \\
\hline Itching & 42 & 84 \\
\hline Scanty Watery Discharge & 34 & 68 \\
\hline Blocking Sensation & 40 & 80 \\
\hline Tinnitus & 36 & 72 \\
\hline Table 4. Symptomatology in Patients with Otomycosis \\
\hline
\end{tabular}

The most common otoscopic findings were blackish (or) brownish white debris followed by greyish white debris, whitish flakes and yellowish debris in that order of frequency.

Table 5 shows the various otoscopic findings of the patients included in our study.

\begin{tabular}{|c|c|c|}
\hline Findings in External Auditory Canal & No. of Ears & $\%$ \\
\hline Black (or) Brownish White Debris & 20 & 40 \\
\hline Greyish White Debris & 16 & 32 \\
\hline Whitish Flakes & 10 & 20 \\
\hline Yellowish Debris & 4 & 8 \\
\hline Table 5. Otoscopy Findings in Patients with Otomycosis \\
\hline
\end{tabular}

The various predisposing factors to otomycosis and their incidence were illustrated in table 6 which was studied as a part of this project.

\begin{tabular}{|c|c|c|}
\hline Predisposing Factors & $\begin{array}{c}\text { No. of } \\
\text { Patients }\end{array}$ & $\mathbf{\%}$ \\
\hline Ear Probing & 42 & 84 \\
\hline Antibiotic and/or Steroid Ear Drops & 24 & 48 \\
\hline Previous Ear Infection & 22 & 44 \\
\hline Allergic Otitis Externa & 10 & 20 \\
\hline Ear Syringing & 8 & 16 \\
\hline Diabetes Mellitus & 3 & 6 \\
\hline Irradiation to Head and Neck & 2 & 4 \\
\hline HIV Infection & 2 & 4 \\
\hline Previous Ear Surgery & 12 & 24 \\
\hline \multicolumn{2}{|c|}{ Table 6. Predisposing Factors } \\
\hline
\end{tabular}

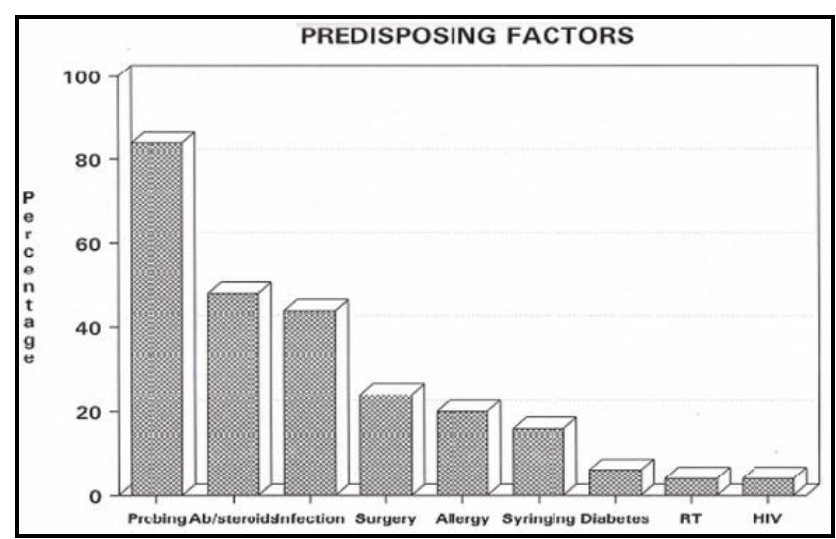

Figure 3. Predisposing Factors

Various Fungal isolates grown in culture were studied. Aspergillus niger was the most common fungi grown in culture followed by Aspergillus flavus, candida, Mucor in the descending order. Two patients had Cephalosporium which was an uncommon finding.

\begin{tabular}{|c|c|c|}
\hline Fungal Isolates & No. of Patients & \% \\
\hline Aspergillus Niger & 23 & 46 \\
\hline Aspergillus Flavus & 13 & 26 \\
\hline Candida sp & 10 & 20 \\
\hline Mucor sp & 2 & 4 \\
\hline Cephalosporium & 2 & 4 \\
\hline
\end{tabular}

Table 7. Fungal Isolates in 50 Patients of Otomycosis ${ }^{5,6}$

\section{DISCUSSION}

Mycotic lesions of the ear is an ubiquitous occurrence which an otolaryngologist commonly encounters in his day-to-day practice. The spectrum of association of otomycosis ranges from simple itching to life threating leukaemias, lymphomas and HIV infection. Hence, it is imperative that a patient with otomycosis is to be thoroughly examined and investigated by the otolaryngologist.

Mycotic lesion of the ear is encountered with a broad spectrum ranging from the most common Aspergillus species to the uncommon Cephalosporium species, from the harmless candida species to the invasive Mucor and/or Aspergillus species, from simple itching to necrotising fungal infection.

Though the incidence of otomycosis per se has not registered any increase, the last decade saw a huge increase in the incidence of otomycosis due to explosion of its associated conditions such as Diabetes mellitus, HIV infection, radiotherapy for malignancy, ${ }^{7}$ the ever-increasing 
indiscriminate use of antibiotics and the double edged weapon - steroids either by over-the-counter use or by general practitioners has also contributed considerably for the increase in the incidence. Hence, this study on the mycotic lesions of the ear achieves significant proportions.

In our series of patients with otomycosis, the most common presenting symptom was itching of the ear in 42 cases (84\%), followed in descending order by hard of hearing in 40 patients $(80 \%)$, ear pain in 36 patients $(72 \%)$ and discharge from ears in 34 patients (68\%).

According to a study conducted by Ostika and Iouka, itching was the mort predominant symptom in $90 \%$ of the patients, followed by ear pain in $70 \%$ and watery discharge in $65 \%$ of the patients, which almost correlates with our study.

In our series, the most common predisposing factor causing otomycosis was ear probing in 42 cases (84\%) followed by indiscriminate use of antibiotics and steroid ear drops $^{8}$ in 24 cases (48\%). The various other predisposing factors causing otomycosis were pre-existing ear infection in 22 cases (44\%), prior ear surgery mainly as a part of cavity problem in 12 cases (24\%), allergy in 10 cases (20\%), syringing of ear in 8 cases (16\%), diabetes mellitus in 3 cases (6\%), irradiation to tumours of the head and neck region in 2 cases (4\%) and HIV in 2 cases (4\%).

In our study group, 3 had diabetes mellitus. One patient was referred from Diabetology department with complaints of ear block, itching and watery discharge. The most common species in patients with diabetes mellitus was Aspergillus niger in 2 patients (66\%), while 1 patient had Mucor (33\%).

In our series, 2 patients had associated HIV infection proved by ELISA. 1 patient had Aspergillus flavus and the other had candida.

Two patients in our study group were post-irradiation patients with features of bone marrow depression, bilateral otomycosis and oral candidiasis. One patient had candida albicans and the other Aspergillus flavus which were amenable to clotrimazole ear drops.

According to Enweic IB and Iguborti, in their study on prevalence of otomycosis the commonest fungal agent isolated by them was Aspergillus niger in $43.8 \% .{ }^{9}$ Hence, our study almost coincides well with the above study in isolating Aspergillus niger as the most predominant fungal species associated with otomycosis.

Patients were followed up at weekly intervals and their response to treatment with $1 \%$ clotrimazole ${ }^{10}$ was assessed. All the patients except two responded well to clotrimazole ear drops. The patients symptoms regressed and they became asymptomatic within two to three weeks. They were followed up for the recurrence for a further period of three weeks. Their external canals did not show any colonisation of fungus.

Among the two patients who had recurrent and persistent residual otomycosis even after regular aural toileting and application of topical antifungal agent one was suffering from uncontrolled diabetes and HIV infection respectively. Hence, they were referred back to the concerned department for management of their systemic illness.

Maher A, Bassioung A, Moauad MK, Hendawy DS in their experimental evaluation of six antimycotic agents on otomycosis has concluded that clotrimazole $1 \%$ was effective in almost all cases, which correlates well with our study.

\section{CONCLUSION}

The most common offending fungus causing otomycosis in this study was Aspergillus niger (46\%). ${ }^{11}$ Uncommonly isolated fungi in this study includes Cephalosporium \& Mucor spp. (2\%) respectively.

On evaluation of different presenting symptoms of patients with otomycosis, it can be concluded that itching of the affected ears was the most common presenting symptom of otomycosis patients (84\%). Equally important were blocking sensation and pain on the affected side.

This study on otomycosis also evaluated the various predisposing factors that may have caused otomycosis and concludes that ear probing and prolonged use of antibiotics/or steroid ear drops and previous ear infection as the common cause of otomycosis. It can also be concluded that systemic illness also plays an important role in the causation and or recurrence of otomycosis despite proper treatment.

This study also proves that topical $1 \%$ clotrimazole ear drops which is commonly available, cost effective and relatively complication free was effective in most cases of otomycosis (96\%).

\section{This Study Incidentally also put forth the following Suggestions}

Patients with recurrent and resistant otomycosis should be systematically examined to rule out systemic illness.

General practitioners should be educated with emphasis on the varying presenting symptoms of otomycosis, varying appearances of the otomycosis flakes, the importance of aural toileting to clear the debris and the spectrum of sensitivity of commonly used drugs such as clotrimazole.

Otomycosis with its omnipotent presence can be treated and the patients can be relieved of their distress immediately in a cost effective way if only the diagnosis is made properly.

Further studies on the involvement of middle \& inner ear, invasiveness of the fungus and new strains of the often used clotrimazole resistant fungus have to be extensively studied so that the morbidity of this disease can be considerably reduced in the next millennium.

\section{REFERENCES}

[1] Rao RP, Rao R. A Mycologic Study of Otomycosis in a Tertiary care Teaching Hospital in Karnataka, India. International Journal of Contemporary Medical Research 2016;3(7):1918-20.

[2] Abou-halawa AS, Khan MA, AlRobaee AA, et al. Otomycosis with perforated tympanic membrane: self medication with topical antifungal solution versus medicated ear wick. Int J Health Sci (Qassim) 2012;6(1):73-7.

[3] Anwar K, Gohar MS. Otomycosis; clinical features, predisposing factors and treatment implications. Pak J Med Sci 2014;30(3):564-7.

[4] Ballenger JJ. Diseases of the nose, throat, ear, head and neck. 14th edn. Lea \& Febiger 1991.

[5] Donamayor HC. Ear infections by Aspergillus. An Otorrinolaringol Ibero Am 1990;17(5):535-44.

[6] Friedmann I, Bennett MH, Piris J. Nose throat, and ears. 3rd edn. Vol. 1. Churchill Livingstone 1986:256-7. 


\section{Jemds.com}

[7] Prakash SB, Leelatejaswini RM, Deekshita V. A clinical and microbial study of otomycosis: an original study. JEMDS 2015;4(71):12376-84.

[8] Prasad SC, Kotigadde S, Shekhar M, et al. Primary otomycosis in the Indian subcontinent: predisposing factors, microbiology, and classification. International Journal of Microbiology 2014;2014:1-9.

[9] Ray R. Pal S, Ghosh M, et al. Prevalence of fungal infection in chronic otitis media-a study at a tertiary
Original Research Article

care hospital in eastern India. IJCMAS 2015;4(3):68490.

[10] Tisher J, Millan J, Rivas P, et al. Study of otomycosis. Source: Acta Otorhinologica Espenola 1995;46(2):85-9.

[11] Viswanatha B, Naseeruddin K. Fungal infections of the ear in immunocompromised host: a review. Mediterr J Hematol Infect Dis 2011;3(1):e2011003. 